(n)

\title{
Breast cancer-associated fibroblasts induce epithelial-to-mesenchymal transition in breast cancer cells
}

\author{
Patsy S H Soon ${ }^{1,2,3,4}$, Edward Kim ${ }^{1,2}$, Cindy K Pon ${ }^{1,2}$, Anthony J Gill ${ }^{2,5}$, Katrina Moore ${ }^{2,6}$, \\ Andrew J Spillane ${ }^{2,6}$, Diana E Benn ${ }^{1,2}$ and Robert C Baxter ${ }^{1,2}$ \\ ${ }^{1}$ Hormones and Cancer Group, Kolling Institute of Medical Research, Royal North Shore Hospital, Sydney, \\ New South Wales, Australia \\ ${ }^{2}$ Northern Clinical School, University of Sydney, Sydney, New South Wales, Australia \\ ${ }^{3}$ South Western Sydney Clinical School, University of New South Wales, Sydney, New South Wales, Australia \\ ${ }^{4}$ Department of Surgery, Bankstown Hospital, Bankstown, New South Wales, Australia \\ Departments of ${ }^{5}$ Anatomical Pathology ${ }^{6}$ Endocrine and Oncology Surgery, Royal North Shore Hospital, \\ St Leonards, New South Wales, Australia
}

Correspondence

should be addressed to

P S H Soon

Email

patsy.soon@sydney.edu.au

\begin{abstract}
Cancer-associated fibroblasts (CAFs) play a role in tumour initiation and progression, possibly by inducing epithelial-to-mesenchymal transition (EMT), a series of cellular changes that is known to underlie the process of metastasis. The aim of this study was to determine whether CAFs and surrounding normal breast fibroblasts (NBFs) are able to induce EMT markers and functional changes in breast epithelial cancer cells. Matched pairs of CAFs and NBFs were established from fresh human breast cancer specimens and characterised by assessment of CXCL12 levels, $\alpha$-smooth muscle actin ( $\alpha$-SMA) levels and response to doxorubicin. The fibroblasts were then co-cultured with MCF7 cells. Vimentin and E-cadherin expressions were determined in co-cultured MCF7 cells by immunofluorescence and confocal microscopy as well as by western blotting and quantitative PCR. Co-cultured MCF7 cells were also assessed functionally by invasion assay. CAFs secreted higher levels of CXCL12 and expressed higher levels of $\alpha$-SMA compared with NBFs. CAFs were also less sensitive to doxorubicin as evidenced by less $\mathrm{H} 2 \mathrm{AX}$ phosphorylation and reduced apoptosis on flow cytometric analysis of Annexin V compared with NBFs. When co-cultured with MCF7 cells, there was greater vimentin and less $\mathrm{E}$-cadherin expression as well as greater invasiveness in MCF7 cells co-cultured with CAFs compared with those co-cultured with NBFs. CAFs have the ability to induce a greater degree of EMT in MCF7 cell lines, indicating that CAFs contribute to a more malignant breast cancer phenotype and their role in influencing therapy resistance should therefore be considered when treating breast cancer.
\end{abstract}

Endocrine-Related Cancer (2013) 20, 1-12

\section{Introduction}

Increasing evidence suggests that fibroblasts play a role in cancer initiation and progression (Elenbaas \& Weinberg 2001, Tlsty \& Hein 2001, Bhowmick et al. 2004, Franco et al. 2010). In particular, a subpopulation of fibroblasts, known as cancer-associated fibroblasts (CAFs), is important in tumour growth and progression (Mueller \& Fusenig 2004). These can be distinguished functionally from 'normal' fibroblasts in tissue distal from the tumour site. 
CAFs are believed to originate from several sources including the resident fibroblast population, tumour epithelial cells (through epithelial-to-mesenchymal transition or EMT) and mesenchymal stem cells (Anderberg \& Pietras 2009). Olumi et al. (1999) found that co-culture of non-tumorigenic human prostate epithelial cells with human prostate CAFs, and not normal prostate fibroblasts, dramatically stimulated growth and altered histology of the epithelial cells. Likewise, Hayward et al. (2001) showed that co-culture of the non-tumorigenic prostate cell line BPH-1 with CAFs resulted in permanent malignant transformation of these epithelial cells. The importance of the tumour microenvironment in cancers has also been demonstrated by the ability of a stromal derived signature to accurately predict disease outcome in breast cancer (Wald et al. 2011).

In patients with breast cancer, as in patients with other solid tumours, metastatic disease rather than the primary tumour is the main cause of death (Sporn 1996). EMT, a process in which a normal polarised epithelial cell undergoes multiple biochemical changes to assume a mesenchymal cell phenotype, including acquisition of migratory and invasive properties and the loss of cell-cell adhesion and cell polarity (Kalluri \& Weinberg 2009), is thought to underlie tumour progression and metastasis. Epithelial cells that have undergone EMT to adopt a mesenchymal phenotype are distinguished by a loss of expression of E-cadherin and expression of mesenchymal markers, which include N-cadherin, ZEB1, SIP1 and vimentin. There is also a change in morphology of the cells to a spindle appearance with loss of cohesion (Gregory et al. 2008). In breast cancer, expression of EMT inducers such as Snail and Slug has been found to correlate with higher tumour grade and basal-like subtype, both of which are associated with worse prognosis (Blanco et al. 2002, Storci et al. 2008).

Several studies have shown that tumour cells that have undergone EMT, as evidenced by the expression of mesenchymal markers, tend to be more resistant to chemotherapy (Yang et al. 2006, Kajiyama et al. 2007). Epithelial cell lines from colon cancer that develop resistance to oxaliplatin have been demonstrated to acquire a mesenchymal phenotype that includes spindle appearance, increased separation between the cells and formation of pseudopodia (Yang et al. 2006). Similarly, epithelial cell lines from ovarian cancer that are resistant to paclitaxel also exhibit a mesenchymal morphology (Kajiyama et al. 2007).

Lebret et al. (2007) found that CAFs and fibroblasts from breast reduction specimens were able to induce EMT in PMC42-LA cells, a breast cancer cell line that has stem cell-like properties. Another study found that fibroblasts isolated from the interface zone located within $5 \mathrm{~mm}$ of the cancer as well as CAFs and normal breast fibroblasts (NBFs) were able to induce EMT in MCF7 cells (Gao et al. 2010). In addition, fibroblasts from benign hypertrophic prostates that have been activated by co-culture with aggressive prostate cancer cell lines demonstrate the ability to induce EMT in a prostate cancer cell line (Giannoni et al. 2010).

The aim of this study was to determine whether CAFs and NBFs respond differently to doxorubicin, a chemotherapeutic agent used frequently in the treatment of breast cancer patients, and to determine whether CAFs and NBFs are able to induce EMT markers in a breast epithelial cancer cell line and a phenotypically normal breast cell line as well as functional changes in the breast epithelial cancer cells.

\section{Materials and methods}

\section{Patients and tumours}

Ethics approval for the study was obtained from the Northern Sydney and Central Coast Area Health Service Human Research Ethics Committee, Sydney, Australia. Before sample collection, informed consent was obtained from patients after full explanation of the purpose and nature of all procedures.

\section{Isolation of fibroblasts}

Matched pairs of CAFs and NBFs were established from fresh human breast cancer specimens. For CAFs, tissue from within the macroscopic tumour was cultured, while for NBFs, tissue from at least $2 \mathrm{~cm}$ away from the macroscopic tumour margin was used. Tissue samples were collected in F-12 medium (Invitrogen) supplemented with 10\% fetal bovine serum (FBS; Invitrogen), penicillinstreptomycin (Invitrogen), $50 \mu \mathrm{g} / \mathrm{ml}$ gentamicin (Invitrogen) and $2.5 \mu \mathrm{g} / \mathrm{ml}$ fungizone (Invitrogen). The tissue was minced and digested overnight at $37^{\circ} \mathrm{C}$ with rotation in medium containing $0.5 \mathrm{mg} / \mathrm{ml}$ collagenase (SigmaAldrich, Inc.). Cells were pelleted by centrifugation, resuspended in buffer containing $0.15 \mathrm{M} \mathrm{NH}_{4} \mathrm{Cl}, 1 \mathrm{M}$ $\mathrm{KHCO}_{3}$ and $0.1 \mathrm{M}$ EDTA, pH 7.23, and incubated for 5 min to lyse red blood cells. After centrifugation, cells were passed through a $100 \mu \mathrm{m}$ filter, followed by a $40 \mu \mathrm{m}$ filter and cultured in DMEM (Invitrogen) supplemented with $10 \%$ FBS, penicillin-streptomycin and $50 \mu \mathrm{g} / \mathrm{ml}$ gentamicin for 1-2 weeks. Fibroblasts were separated from epithelial cells by differential trypsinisation.

Published by Bioscientifica Ltd. 
The cells were trypsinised for $5 \mathrm{~min}$ at $37^{\circ} \mathrm{C}$ - fibroblasts that are more sensitive to trypsin were detached from the flask while epithelial cells remained attached.

\section{Pathological review}

Representative haematoxylin- and eosin (H\&E)-stained slides of each cancer and of benign breast tissue from more than $2 \mathrm{~cm}$ away from the macroscopic lesion (corresponding as closely as possible to the sampled areas) were independently reviewed by a single pathologist (A J G) who was blinded to all other data. The pathologist classified and graded all tumours using the WHO 2012 system (Lakhani et al. 2012) and staged the tumours using the AJCC 7th edition 2009 staging system (Edge et al. 2009). Based on this morphologic review, the pathologist estimated the percentage of each cell type (invasive carcinoma, in situ carcinoma, non-adipose stromal cells (fibroblasts and myofibroblasts), adipose cells and lymphocytes) in the two sampled areas in 5\% increments.

\section{Cell culture}

Primary fibroblasts isolated from patient tissue were maintained in DMEM containing $10 \%$ FBS at $37^{\circ} \mathrm{C}$ in a humidified incubator containing 5\% $\mathrm{CO}_{2}$. The human breast cancer cell line, MCF7 (American Type Culture Collection (ATCC), Manassas, VA, USA), was maintained in Roswell Park Memorial Institute (RPMI) 1640 medium containing 10\% FBS. The MCF7 cells were authenticated using short tandem repeat profiling at Cellbank Australia (Sydney, NSW, Australia). Out of nine loci, 14/14 alleles were identical to the original MCF7 profile. MCF10A breast epithelial cells (ATCC) were cultured in DMEM/F12 medium (Invitrogen) containing 5\% horse serum (Invitrogen), $10 \mu \mathrm{g} / \mathrm{ml}$ insulin (Sigma-Aldrich, Inc.), $10 \mathrm{ng} / \mathrm{ml}$ EGF (Sigma-Aldrich, Inc.) and $0.5 \mu \mathrm{g} / \mathrm{ml}$ hydrocortisone (Sigma-Aldrich, Inc.). Primary cells used for experiments were passaged to a maximum of six passages, with experiments performed on passage 5 and 6 cells.

\section{Conditioned media experiments}

Medium conditioned for $72 \mathrm{~h}$ by primary fibroblasts grown in DMEM containing 10\% FBS was collected. MCF7 or MCF10A cells were seeded in six-well plates (Nunc, New York City, NY, USA) at $1 \times 10^{5}$ cells/well. After $24 \mathrm{~h}$, cells were treated with a 1:1 mixture of medium conditioned by fibroblasts and fresh RPMI medium with $10 \%$ FBS for 6 or 10 days.

\section{Indirect co-culture of fibroblasts with cancer cells}

MCF7 cells $\left(1.5 \times 10^{4}\right)$ were seeded on a 12-well plate in $1 \mathrm{ml}$ RPMI with $10 \% \mathrm{FBS}$ and $1.5 \times 10^{4}$ fibroblasts were seeded on the $0.4 \mu \mathrm{m}$ polyester membrane of a $12 \mathrm{~mm}$ transwell insert (Corning, Lowell, MA, USA) in $1 \mathrm{ml}$ DMEM with $10 \%$ FBS and placed in a separate culture plate. The transwell insert was subsequently transferred to the culture plate with MCF7 cells the next day and cells were cultured in $1 \mathrm{ml}$ RPMI/DMEM with $10 \%$ FBS for 6 days. Medium was replenished after 3 days of co-culture.

\section{Immunofluorescence}

MCF7 cells were seeded on chamber slides $\left(1 \times 10^{5}\right.$ cells $)$ and allowed to adhere for $48 \mathrm{~h}$. Cells were then treated with a 1:1 mixture of medium conditioned for $72 \mathrm{~h}$ by fibroblasts and RPMI medium for 3 and 6 days. Control cells were treated with 1:1 mixture of RPMI and unconditioned DMEM. The cells were washed with PBS, fixed in $4 \%$ paraformaldehyde for $20 \mathrm{~min}$ at room temperature (RT), washed with cold PBS and incubated with $0.1 \%$ Triton X-100 at RT for $10 \mathrm{~min}$. Cells were incubated with $10 \%$ normal goat serum at RT for $10 \mathrm{~min}$, primary antibody (E-cadherin \#AB1416 and vimentin \#AB8069, Abcam, Cambridge, UK, at 1:250 dilution) at RT for $1 \mathrm{~h}$ and secondary antibody (anti-mouse Alexa fluor 488, Invitrogen, at 1:200 dilution) at RT for $1 \mathrm{~h}$. Excess antibody was removed by washing the cells in PBS three times. Cells were stained with DAPI $(1 \mu \mathrm{g} / \mathrm{ml})$ and then Vectashield (Vector, Burlingame, CA, USA) was used as a mounting medium. Staining was assessed by fluorescence microscopy or confocal microscopy. For fluorescence microscopy, images were captured on an Olympus BX51 microscope (Tokyo, Japan) using the Olympus Micro DP Controller program and DP Manager was used to overlay the images. For confocal microscopy, images were captured on a Leica SP5 confocal microscope (Leica Microsystems, Wetzlar, Germany) using the Las AF software (Leica Microsystems). Quantitation of staining was performed using Adobe Photoshop CS5 (Adobe Systems, Inc.).

\section{Quantitative RT-PCR}

RNA was extracted using TRI reagent (Sigma-Aldrich, Inc.). Purified RNA ( $1 \mu \mathrm{g})$ was reverse-transcribed using Superscript III First-Strand Synthesis Supermix kit (Invitrogen) in a total volume of $20 \mu \mathrm{l}$ according to manufacturer's instructions. The TaqMan probes used in this study were CDH1 (Assay ID \#HS01023894_M1) and

Published by Bioscientifica Ltd 
vimentin (Assay ID \#HS00185584_M1). 18S (Assay ID \#HS99999901_S1) was used as a housekeeping gene. Quantitative RT-PCRs (qPCRs) for each sample were performed in triplicate in two independent experiments. Reactions for qRT-PCR were set up using an Eppendorf epMotion5070 robotic system (Eppendorf, Hamburg, Germany) and performed on the Rotor-Gene RG-3000 real-time rotary analyser (Corbett Research, Mortlake, NSW, Australia). Cycle thresholds were chosen manually on the Rotor-Gene 6 Analysis Software. Differences between groups were assessed statistically using RESTXL@-version 2 (Relative Expression Software Tool; Qiagen; Brabletz et al. 2005) where relative expression ratios are computed based on the PCR efficiency and crossing point differences.

\section{Western immunoblotting}

Cells were lysed in NuPAGE sample buffer, sonicated and equal volumes were separated by SDS-PAGE on $4-12 \%$ NuPAGE Bis-Tris gels (Invitrogen) under reducing conditions. Proteins were transferred to nitrocellulose membrane (GE Healthcare, Little Chalfont, Buckinghamshire, UK) using an XCell Blot Module (Invitrogen). Membranes were blocked in 5\% skim milk powder (Diploma, Mount Waverley, VIC, Australia) dissolved in TBST (TBS/0.1\% Tween 20) for $2 \mathrm{~h}$ at RT and then incubated with the relevant primary antibody according to the manufacturer's instructions. Immunoblots were washed three times with TBST for 5-10 min and incubated with the relevant secondary antibody conjugated to HRP. Blots were then washed three times again in TBST for 5-10 min and incubated with the ECL Plus Western Blotting Detection Reagent (GE Healthcare). The LAS-3000 system was used to capture the chemiluminescent signal (Fujifilm, Brookvale, NSW, Australia). Quantitation was performed using Multi-Gauge 3.11 software (Fujifilm).

\section{ELISA}

Fibroblasts were seeded at a density of $1 \times 10^{5}$ cells/well in six-well culture plates (Nunc) and grown in serum-free DMEM medium for $72 \mathrm{~h}$. Conditioned medium was collected and concentrated 50-fold using 5K MWCO concentrators (Agilent Technologies, Inc., Santa Clara, CA, USA). One hundred microlitres of concentrated conditioned medium were assayed for CXCL12 using a CXCL12 ELISA kit (R\&D Systems, Minneapolis, MN, USA) following manufacturer's instructions. Any value of the concentrated conditioned medium that was less than half of the lowest standard $(16 \mathrm{pg} / \mathrm{ml})$ was assigned a value of zero before data analysis.

\section{Flow cytometry}

Fibroblasts were seeded at $1 \times 10^{5}$ cells/well in 24 -well tissue culture plates and allowed to adhere overnight. Cells were then treated with vehicle, 0.5 or $1 \mu \mathrm{M}$ doxorubicin for $6 \mathrm{~h}$ in serum-free medium. Medium was removed and cells were washed with PBS. Cells were then harvested using citric saline, washed once in PBS and washed in Annexin V binding buffer (BioLegend, San Diego, CA, USA). The cell pellet was resuspended in $100 \mu \mathrm{l}$ Annexin $\mathrm{V}$ binding buffer, $5 \mu \mathrm{l}$ Alexa Fluor 647 Annexin $\mathrm{V}$ and $10 \mu \mathrm{l}$ $1 \mathrm{mg} / \mathrm{ml}$ propidium iodide (Sigma-Aldrich, Inc.) was added to each sample. Cells were subjected to gentle vortexing and were incubated with the dyes for $15 \mathrm{~min}$ at $\mathrm{RT}$ in the dark.

Five hundred microlitres of Annexin $\mathrm{V}$ binding buffer were added to each sample and analysed by flow cytometry using the BD FACSCalibur (BD Biosciences, CA, USA) and CellQuest Pro software (BD Biosciences).

\section{Cell proliferation assay}

MCF7 cells were seeded into 24 -well plates at $1 \times 10^{5}$ cells/well and allowed to adhere overnight. The cells were

Table 1 Patient demographics and pathology results of breast cancer samples used for culturing of CAFs and NBFs.

\begin{tabular}{|c|c|c|c|c|c|c|c|c|c|c|c|}
\hline $\begin{array}{l}\text { CAF/NBF } \\
\text { cell line }\end{array}$ & Age & Side & Operation & $\begin{array}{l}\text { Type of } \\
\text { cancer }\end{array}$ & Grade & $\begin{array}{l}\text { Size } \\
(\mathrm{mm})\end{array}$ & Stage & ER & PR & HER2 & $\begin{array}{c}\text { HER2 mean copy } \\
\text { number }\end{array}$ \\
\hline 2 & 82 & Left & WLE/ANC & Ductal & 1 & 25 & $\mathrm{p} 2 \mathrm{~A}(\mathrm{~T} 2 \mathrm{~N} 0)$ & + & + & - & 2.15 \\
\hline 3 & 67 & Left & Mastectomy/ANC & Lobular & 2 & 17 & $\mathrm{p} 2 \mathrm{~A}(\mathrm{~T} 1 \mathrm{~N} 1)$ & + & - & - & 1.42 \\
\hline 6 & 80 & Right & Mastectomy/ANC & Ductal & 3 & 40 & p2B(T2N1) & + & + & + & 9.12 \\
\hline 7 & 41 & Left & WLE/ANC & Ductal & 3 & 22 & $\mathrm{p} 2 \mathrm{~B}(\mathrm{~T} 2 \mathrm{~N} 1)$ & + & + & - & 1.81 \\
\hline 10 & 60 & Left & WLE/SLNB & Ductal & 1 & 18 & $\mathrm{p} 2 \mathrm{~A}(\mathrm{~T} 1 \mathrm{CN} 0)$ & + & + & - & 1.5 \\
\hline
\end{tabular}

WLE, wide local excision; ANC, axillary node clearance; SLNB, sentinel lymph node biopsy; ER, oestrogen receptor; PR, progesterone receptor; HER2, human epidermal growth factor receptor type 2 .

http://www.erc.endocrinology-journals.org DOI: $10.1530 /$ ERC-12-0227
(C) 2013 Society for Endocrinology Printed in Great Britain
Published by Bioscientifica Ltd 
then incubated with a 1:1 mixture of medium conditioned by fibroblasts and fresh RPMI medium with 10\% FBS for 3 days. Cells were harvested by trypsinisation and viable cells were counted using Trypan blue exclusion.

\section{Invasion assay}

MCF7 cells were cultured in control or conditioned medium from fibroblasts for 6 days. Cells were then harvested, washed twice in PBS and seeded at a density of $2 \times 10^{5}$ cells/well in the transwell of BD biocoat growth factor-reduced matrigel invasion chamber plates (BD Biosciences, Franklyn Lakes, NJ, USA) in their respective treatment medium containing 5\% FBS and $5 \mu \mathrm{M}$ of cytosine $\beta$-D-arabinofuranoside (Sigma-Aldrich, Inc.). Treatment medium containing $15 \%$ FBS and $5 \mu \mathrm{M}$ cytosine $\beta$-D-arabinofuranoside (Sigma-Aldrich, Inc.) was used in the lower chamber. Cells were given $48 \mathrm{~h}$ to invade through matrigel. Medium and cells within the transwells were removed with cotton swabs. Transwells were placed in methanol for $20 \mathrm{~min}$ at $-20^{\circ} \mathrm{C}$ to fix cells to the membrane, washed with PBS and dried. Membranes were excised from the transwells and mounted onto slides using Prolong antifade gold reagent with DAPI (Invitrogen). Staining was assessed by fluorescence microscopy where images were captured on the Olympus BX51 microscope. Cells were counted using Image J 1.45S (NIH, Bethesda, MD, USA).

\section{Statistical analysis}

Experiments were generally performed in triplicate at least three times unless otherwise stated. Statistical analysis of the data was performed using ANOVA or repeated measures ANOVA where appropriate. Differences between groups were evaluated by the Fisher's protected least

A
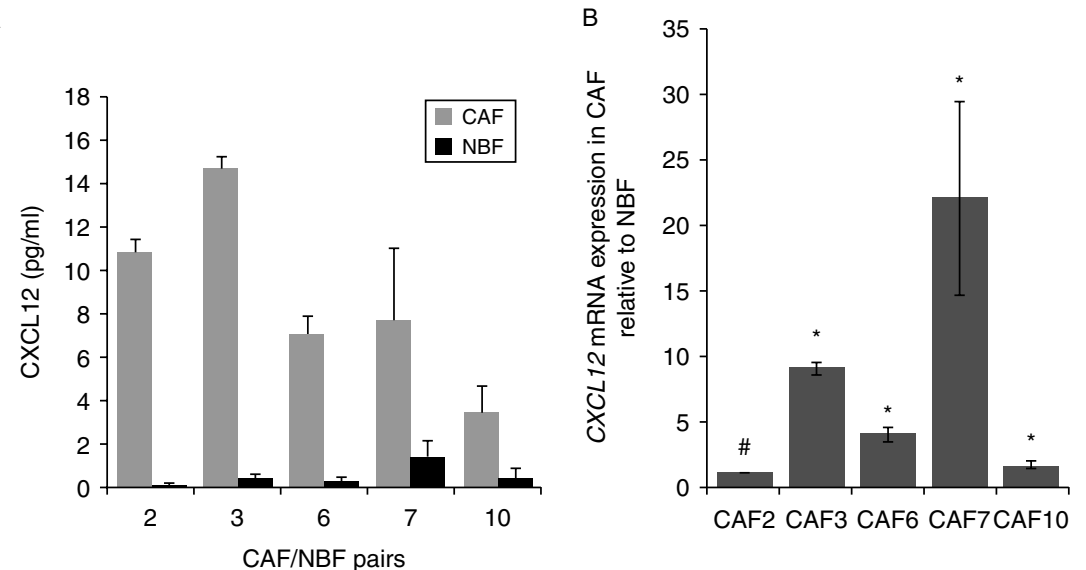

C

D

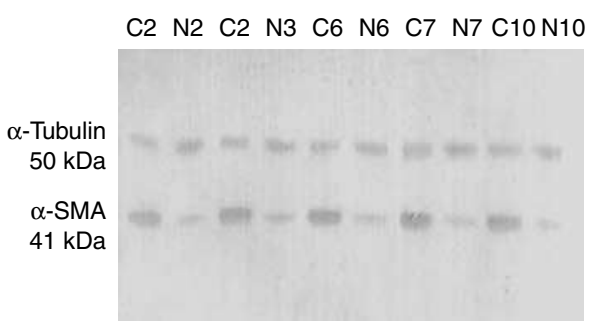

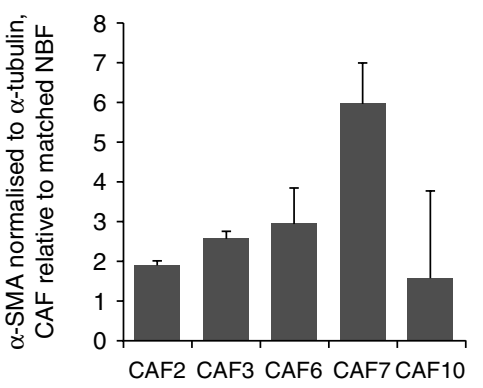

\section{Figure 1}

Characterisation of CAFs and NBFs. (A) CXCL12 levels as measured by ELISA in matched CAFs and NBFs. CAFs secreted an average of $30 \pm 14$-fold higher amounts of $\mathrm{CXCL} 12$ compared with corresponding NBFs $(P=0.013)$. Results are average of two experiments done in triplicate. Error bars indicate S.E.M. (B) CXCL12 mRNA expression by qPCR in matched CAFs and NBFs. CAFs expressed on average $7.7 \pm 3.9$ times more $C X C L 12$ mRNA compared with matched NBFs. $P<0.05$ for four of the five pairs, marked as *. $P=0.29$ for CAF/NBF2, marked as \#. Results are average of two experiments performed in duplicate. Error bars indicate S.E.M. (C) Western blot of $\alpha$-SMA expression in matched CAFs and NBFs. Representative western blot of $\alpha$-SMA (41 kDa) and $\alpha$-tubulin ( $50 \mathrm{kDa}$ ) in matched CAFs and NBFs showing higher expression of $\alpha$-SMA in CAFs compared with matched NBFs. (D) Fold change of $\alpha$-SMA expression in matched CAFs and NBFs, normalised to $\alpha$-tubulin. CAFs express on average $3.0 \pm 0.8$-fold more $\alpha$-SMA compared with matched NBFs $(P<0.001)$. Results are average of three experiments. Error bars indicate s.E.M. http://www.erc.endocrinology-journals.org DOI: 10.1530/ERC-12-0227
(C) 2013 Society for Endocrinology Printed in Great Britain 
significant difference (PLSD) test and results were considered significant if $P<0.05$. The statistical package StatView for Windows Version 5.0 (SAS Institute, Inc., Cary, NC, USA) was used. Results are expressed as mean \pm s.E.m. unless otherwise stated.

\section{Results}

Five matched pairs of CAFs and NBFs were established from fresh human breast cancer specimens. The patient demographics and pathology details of the breast cancer samples are shown in Table 1.

\section{Pathological review}

All the carcinomas were of pathologic stage p2A or p2B. Four of the carcinomas were of invasive ductal type, whereas one of the carcinomas was of invasive lobular type. All the blocks sampled as cancer contained invasive carcinoma cells accounting for from 50 to $75 \%$ of cells as well as non-adipose stromal cells accounting for between 10 and $15 \%$ of cells with few adipose cells (10\% or less). None of the areas sampled as benign tissue contained invasive malignancy, although one case did contain lobular carcinoma in situ (5\%). The epithelial cells in these areas accounted for between 35 and $45 \%$ of all cells, with the percentage of non-adipose stromal cells (35-45\%) and adipose cells (10-25\%) being significantly higher than seen in the areas sampled as cancer. The benign areas did include occasional foci of simple hyperplasia without atypia but were otherwise normal. In particular, there were no areas of atypical ductal hyperplasia or morphologically discernible stromal abnormalities.
A

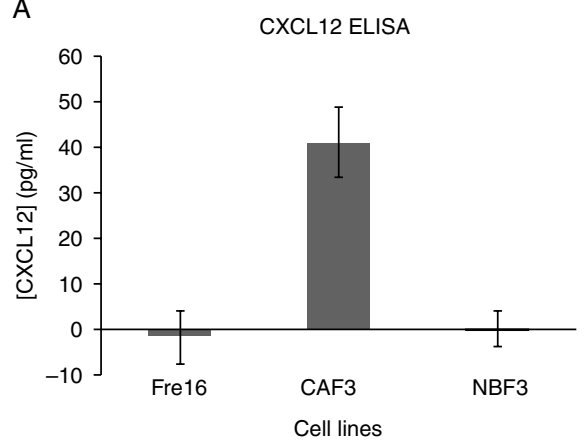

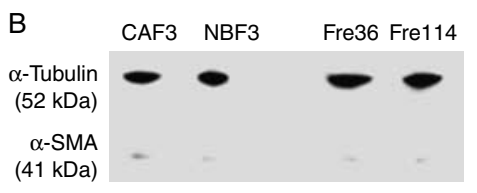

$\alpha-S M A / \alpha$-Tubulin

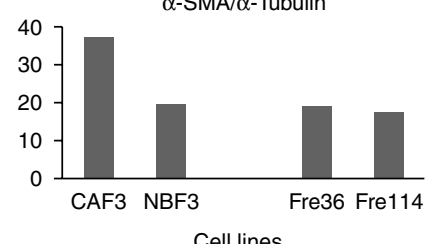

Cell lines

C
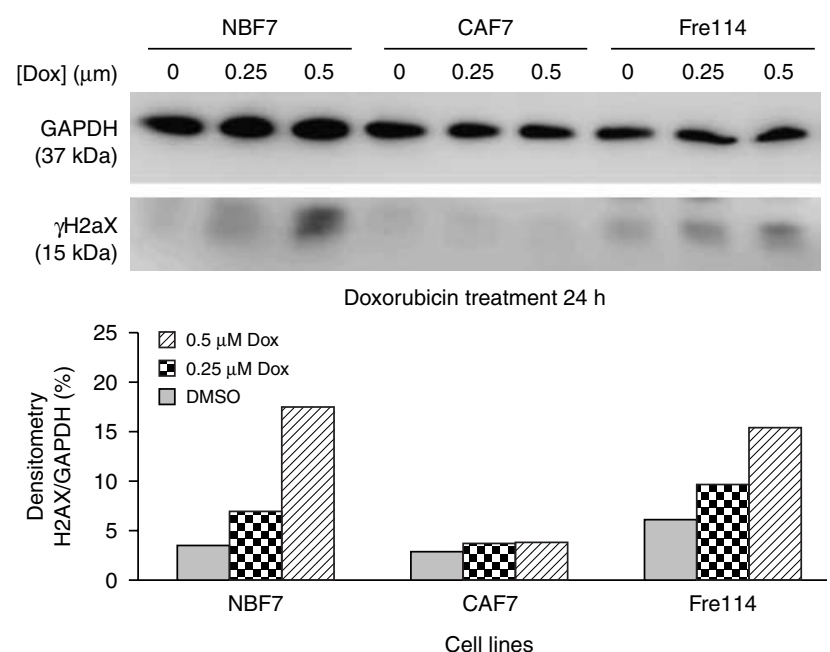

Figure 2

Fibroblasts from breast reduction specimens (Fre) compared with CAFs and NBFs. (A) CXCL12 levels by ELISA. CAFs expressed higher CXCL12 levels by ELISA compared with NBFs and Fre. (B) Western blot and densitometry of $\alpha$-smooth muscle actin ( $\alpha$-SMA) and $\alpha$-tubulin expression in CAF, NBF and Fre. CAFs expressed higher levels of $\alpha$-SMA compared with NBFs and Fre,
(C) 2013 Society for Endocrinology Printed in Great Britain which expressed similar levels. (C) Western blot and densitometry of $\gamma \mathrm{H} 2 \mathrm{AX}$ and GAPDH in CAF, NBF and Fre, which was exposed to $0,0.25$ and $0.5 \mu \mathrm{M}$ doxorubicin for $24 \mathrm{~h}$. Fre expressed higher levels of $\mathrm{H} 2 \mathrm{AX}$ in response to doxorubicin treatment compared with CAFs but comparable with NBFs. Experiments were performed in duplicate three times. 

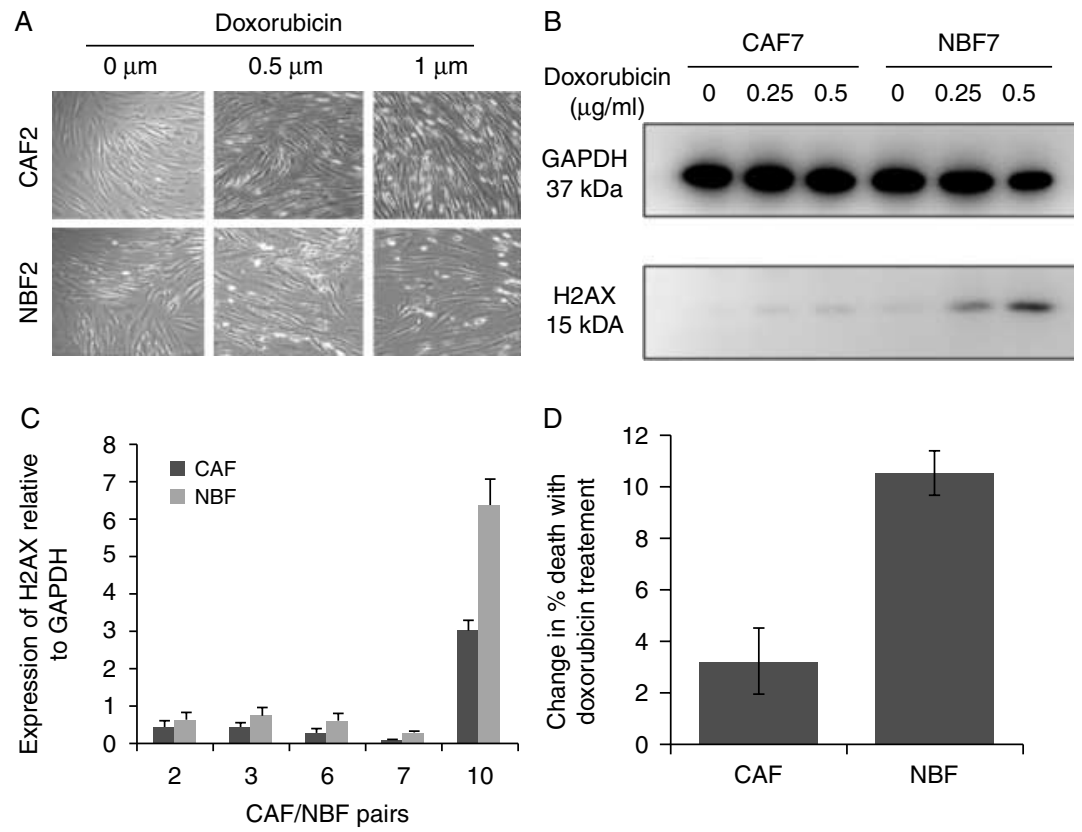

\section{Figure 3}

$\gamma \mathrm{H} 2 \mathrm{AX}$ phosphorylation and Annexin $\mathrm{V}$ by flow cytometry in CAFs and NBFs treated with doxorubicin. (A) Light micrographs of CAF2 and NBF2 at $20 \times$ magnification after no treatment or treatment with 0.5 and $1.0 \mu \mathrm{M}$ doxorubicin for $72 \mathrm{~h}$. (B) A representative western blot of $\gamma \mathrm{H} 2 \mathrm{AX}$ and GAPDH in cell lysates of CAF7 and NBF7 cells treated with $0,0.25$ and $0.5 \mu \mathrm{M}$ doxorubicin. (C) Densitometry results of western blots of $\gamma \mathrm{H} 2 \mathrm{AX}$ normalised to GAPDH in matched CAFs and NBFs treated with $0.5 \mu \mathrm{M}$ doxorubicin. Results are average of experiments performed in triplicates

\section{CAFs and NBFs differ in CXCL12 secretion and $\alpha$-SMA expression}

For initial characterisation of CAF and NBF cultures, we measured secretion of the chemokine CXCL12, also known as stromal derived factor-1 (SDF1), and cellular expression of $\alpha$-smooth muscle actin ( $\alpha$-SMA). These factors may be regarded as two of the hallmarks of CAFs (Mishra et al. 2008). As shown in Fig. 1A, CAFs secrete a mean of $30 \pm 14$-fold (range: 5.3 - to 82.5 -fold) higher amounts of CXCL12, measured by ELISA, compared with corresponding NBFs $(P=0.013)$. By qPCR (Fig. 1B), CAFs express on average $7.7 \pm 3.9$-fold (range: 1.1 - to 22.2 -fold) more CXCL12 mRNA compared with matched NBFs $(P<0.0001)$. While both CAFs and NBFs stain for $\alpha$-SMA by immunocytochemistry, CAFs express 3.0 \pm 0.8 times more $\alpha$-SMA than corresponding NBFs $(P=0.003)$ on western blotting (Fig. $1 \mathrm{C}$ and D). We also found that the level of CXCL12 secretion (Fig. 2A) and $\alpha$-SMA expression (Fig. 2B) in NBFs were similar to fibroblasts cultured from breast reduction specimens.

twice. Error bars indicate S.E.M. There is significantly higher $\gamma \mathrm{H} 2 \mathrm{AX}$ levels in NBFs compared with matched CAFs $(P=0.007)$, indicating a higher level of DNA damage in response to doxorubicin. (D) Annexin $V$ flow cytometry results in two pairs of matched CAFs and NBFs (CAF/NBF6 and CAF/NBF7). Results are average of two experiments done in triplicate. Error bars indicate S.E.M. There are 3.3 times more dead cells in NBFs compared with CAFs treated with doxorubicin $(P=0.0019)$.

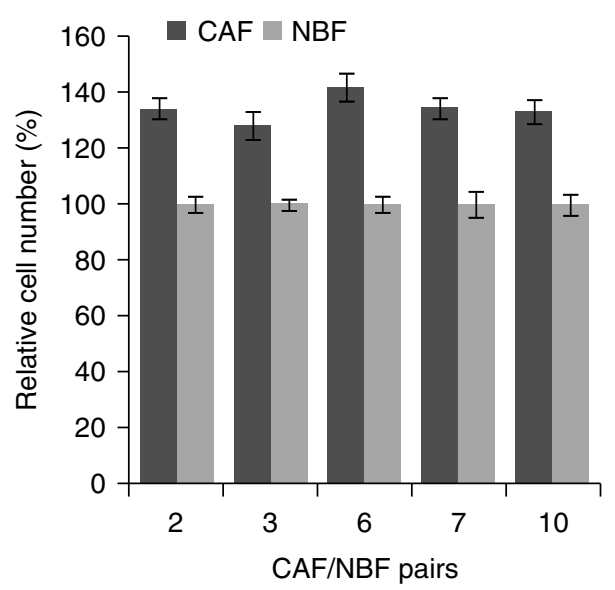

Figure 4

Percent increase in MCF7 cell count grown in CAF- compared with NBF-conditioned media after 3 days. On average, there was a $34 \pm 6 \%$ increase in MCF7 cells grown in CAF-conditioned media compared with NBF-conditioned media after 3 days $(P<0.0001)$.

Published by Bioscientifica Ltd. 

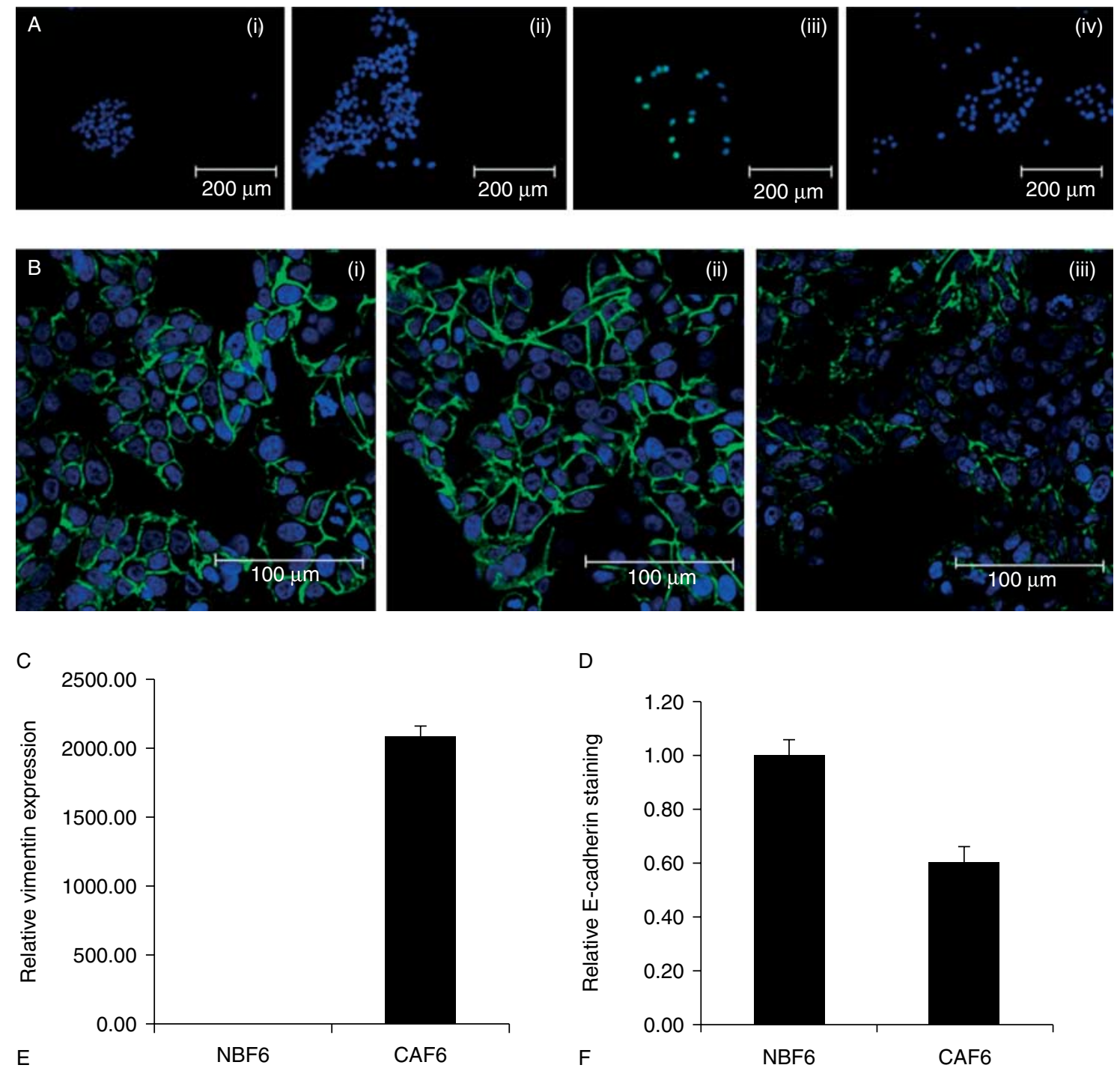

D
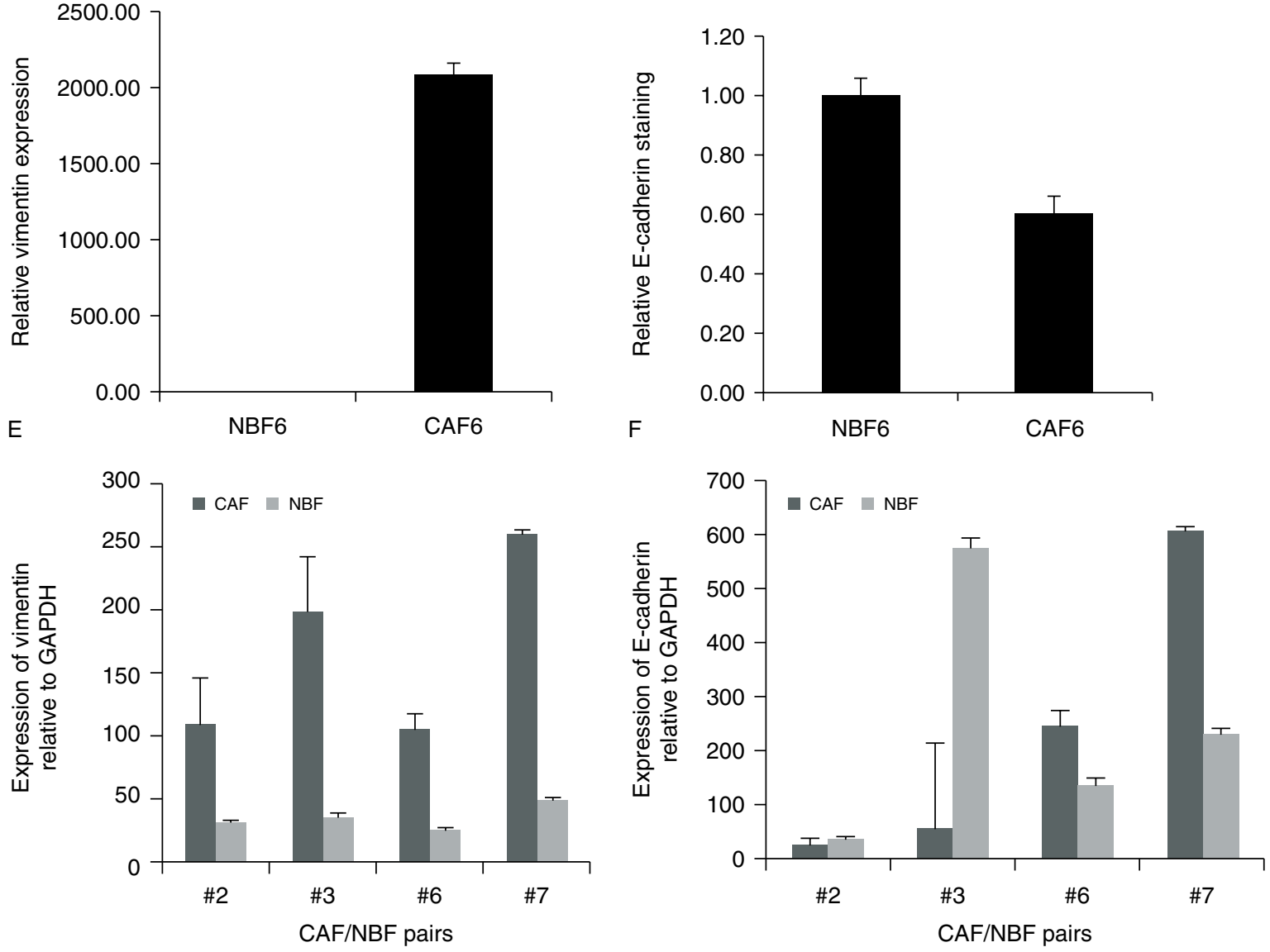


\section{CAFs are more resistant to chemotherapy}

To test the chemoresistance of the CAF cultures, the response of CAFs and NBFs to doxorubicin, a chemotherapeutic agent that is commonly used to treat breast cancer patients, was also assessed. Figure 3A shows morphological changes in one of the matched CAF/NBF cell line pairs in response to 0.5 and $1 \mu \mathrm{M}$ doxorubicin after $72 \mathrm{~h}$. In response to the higher doxorubicin dose, the NBFs showed a greater loss of cells compared with the CAFs, which appeared morphologically unchanged from control cultures. The response of these cells to doxorubicin was quantitated using a marker of the induction of DNA double-stranded breaks, the phosphorylation of histone H2AX ( $\gamma \mathrm{H} 2 \mathrm{AX}$; Fig. 3B and C) as well as Annexin V quantitation on flow cytometry (Fig. 3D). Averaged over five pairs of cultures, CAFs had $51 \pm 47 \%$ lower $\gamma \mathrm{H} 2 \mathrm{AX}$ in response to doxorubicin compared with NBFs $(P=0.007$; Fig. 3C). On Annexin $\mathrm{V}$ analysis by flow cytometry on two pairs of CAF/NBFs (CAF/NBF 6 and 7; Fig. 3D), there were 3.3 times more dead NBFs compared with CAFs after treatment with $1 \mu \mathrm{M}$ doxorubicin $(P=0.0019)$. We also noted that the expression of $\gamma \mathrm{H} 2 \mathrm{AX}$ after treatment with doxorubicin was higher in fibroblasts cultured from breast reduction specimens compared with CAFs but comparable to NBFs (Fig. 2C).

\section{CAFs induce proliferation in MCF7 cells to a greater degree than NBFs}

MCF7 cells cultured in medium conditioned by CAF cell lines, compared with corresponding NBFconditioned media, resulted in $34 \pm 6 \%$ higher MCF7 cell counts $(P<0.0001)$ after 3 days (Fig. 4$)$.

\section{CAFs induce EMT in MCF7 cells to a greater degree than NBFs}

MCF7 cells exposed to culture medium conditioned by CAFs showed stronger staining for vimentin (Fig. 5A) and less for E-cadherin as demonstrated by immunofluorescence and confocal microscopy (Fig. 5B) compared with those exposed to culture medium conditioned by NBFs. Quantitation of staining of vimentin and E-cadherin on confocal microscopy confirmed visual results, showing that MCF7 cells cultured in CAF-conditioned media expressed higher levels of vimentin and lower levels of E-cadherin compared with MCF7 cells cultured in NBFconditioned media (Fig. 5C and D).

There was also more vimentin detectable by western blotting in MCF7 cells cultured in CAF- compared to NBFconditioned medium $(P<0.0001 ;$ Fig. 5E). While the expression of E-cadherin in MCF7 cells exposed to CAFand NBF-conditioned medium for 6 days was variable among the different pairs, E-cadherin detectable by western blotting in MCF7 cells cultured in CAF-conditioned medium was lower compared with cells cultured in NBF-conditioned medium for 10 days (Fig. 5F). We found that there was a consistent significant loss of E-cadherin as measured by western blotting after 10 days $(P=0.0003)$ but not after 6 days $(P=0.67$, data not shown).

\section{CAFs also induce EMT to a greater degree in MCF10A compared with NBFs}

Exposure of CAF- and NBF- conditioned medium to phenotypically normal MCF10A mammary epithelial cells also resulted in an increase in vimentin mRNA measured by qPCR $(P=0.028$; Fig. 6A) and expression of vimentin as measured by western blotting after 6 days $(P<0.007$; Fig. 6B and $C)$.

\section{Invasion studies}

As EMT is known to facilitate cell invasiveness, we examined the effect of conditioned medium studies on MCF7 cells with two pairs of CAFs and corresponding NBFs on their ability to invade. MCF7 cells exposed

\section{Figure 5}

MCF7 cells in CAF- or NBF-conditioned medium experiments. (A) Immunofluorescence for vimentin in (i) negative control (control MCF7 cells with no antibody), (ii) control MCF7 cells in 1:1 RPMI and unconditioned DMEM medium, (iii) MCF7 cells in CAF2 conditioned medium and (iv) MCF7 cells in NBF2 conditioned medium. Blue stain is DAPI staining of the nucleus while green stain is vimentin staining. (B) Confocal microscopy pictures for E-cadherin in (i) control MCF7 cells in 1:1 RPMI and unconditioned DMEM medium, (ii) MCF7 cells in NBF-conditioned medium and (iii) MCF7 cells in CAF-conditioned medium. Blue stain is DAPI staining of the nucleus while green stain is E-cadherin staining. (C) Quantitation of confocal microscopy photomicrographs for expression of E-cadherin. There were lower levels of E-cadherin expressed in MCF7 cells in CAF-conditioned

http://www.erc.endocrinology-journals.org DOI: 10.1530/ERC-12-0227
(C) 2013 Society for Endocrinology Printed in Great Britain media compared with cells in NBF-conditioned media. (D) Quantitation of confocal microscopy photomicrographs for expression of vimentin. There were higher levels of vimentin expressed in MCF7 cells in CAF-conditioned media compared with cells in NBF-conditioned media. (E) E-cadherin expression by western blot in MCF7 cells in CAF- or NBF-conditioned media for 10 days. E-cadherin expression was significantly lower in MCF7 cells in CAF-conditioned media compared with MCF7 cells in NBF-conditioned media $(P=0.0003)$. (F) Vimentin expression by western blot in MCF7 cells grown in CAF- or NBF-conditioned media for 6 days. Vimentin expression was significantly higher in MCF7 cells in CAF-conditioned media compared with MCF7 cells in NBF-conditioned media $(P<0.0001)$. 
A
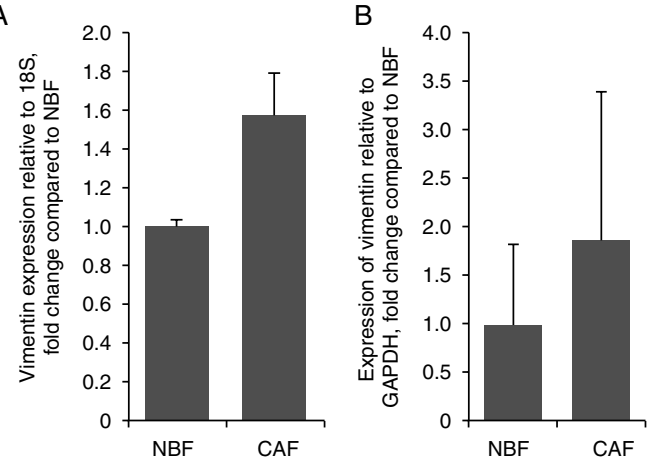

C

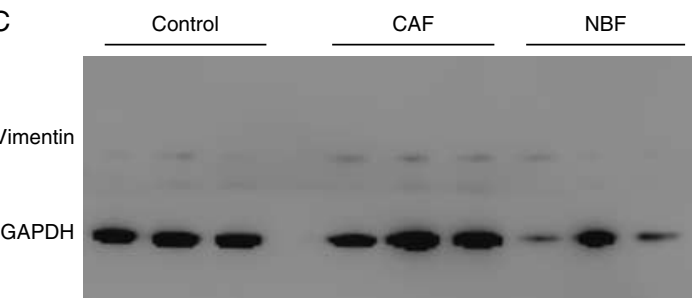

\section{Figure 6}

MCF10A cells grown in medium conditioned by CAFs or NBFs. (A) Expression of vimentin by western blotting was higher in MCF10A cells grown in CAF-conditioned medium for 6 days compared with NBF-conditioned medium $(P=0.007)$. Results are average of three experiments. (B) Expression of vimentin by qPCR was also higher in MCF10A cells grown in CAF-conditioned medium for 6 days compared with NBF-conditioned medium $(P=0.028)$. Results are average of three experiments done in triplicate. (C) Representative western blot of expression of vimentin and GAPDH in control MCF10A cells and MCF10A cells in CAF- or NBF-conditioned media for 6 days.

to CAF-conditioned medium demonstrated greater invasiveness through a matrigel layer compared with cells exposed to NBF-conditioned medium with $P$ value $<0.0021$ (Fig. 7A and B).

\section{Discussion}

In this study, we established five pairs of matched CAFs and NBFs from fresh breast cancer tissue specimens. These two groups of fibroblasts differed in terms of CXCL12 secretion and $\alpha$-SMA expression. While some studies have found that $\alpha$-SMA by immunohistochemistry is only observed in CAFs, not NBFs (Giannoni et al. 2010, Huang et al. 2010), other studies, like ours, have found that $\alpha$-SMA is expressed by both CAFs and NBFs (Lebret et al. 2007, Hawsawi et al. 2008, Holliday et al. 2009), but at higher levels in the CAFs by western blotting (Lebret et al. 2007).

We also found CAFs to be more resistant to doxorubicin compared with matched NBFs. Sonnenberg et al. (2008) assessed sensitivity of CAFs cultured from breast and lung cancers and found that they were less sensitive to paclitaxel and cisplatin compared with 22 cell lines from lung, breast and ovarian cancers as well as acute and chronic myeloid leukaemias. In this study, however, comparison was not made to normal fibroblasts. In response to doxorubicin, we found less DNA doublestranded breaks, as measured by $\gamma \mathrm{H} 2 \mathrm{AX}$, and less apoptosis, as measured by Annexin $\mathrm{V}$ by flow cytometry in CAFs when compared with matched NBFs. The resistance of CAFs to chemotherapy may contribute to the resistance of some cancers to chemotherapy with resultant development of recurrence or metastasis after chemotherapy.

In addition, we have demonstrated that CAFs are able to induce EMT to a greater degree in MCF7 breast cancer cells and phenotypically normal MCF10A breast epithelial cells compared with matched NBFs, as demonstrated by increased expression of vimentin on western blotting. For measurement of E-cadherin by western blotting, we found that the expression was variable after 6 days of co-culture of fibroblasts with MCF7 cells, with no consistent changes observed. After 10 days of co-culture of fibroblasts with
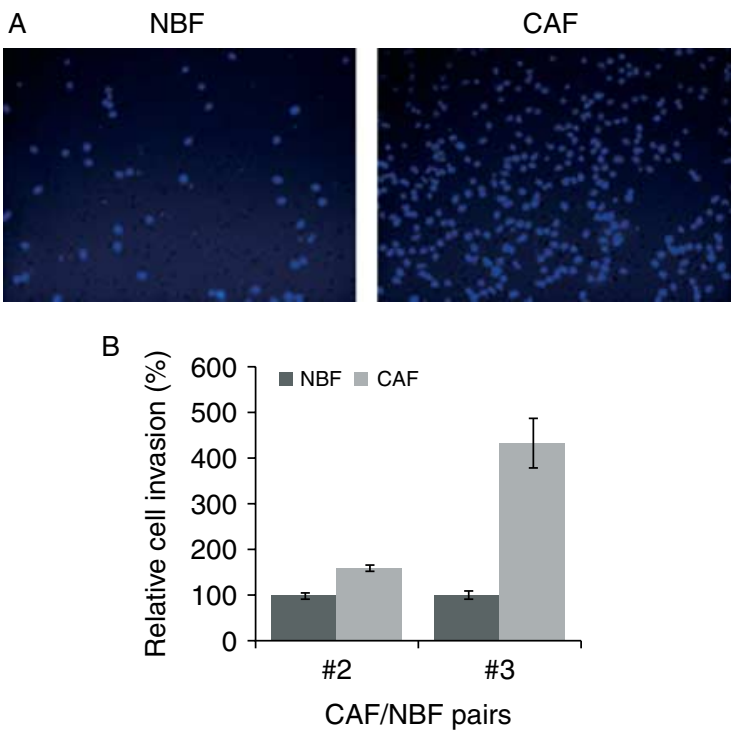

Figure 7

Invasion assay of MCF7 cells in medium conditioned either by CAFs or NBFs. (A) Representative fluorescence photomicrograph of invasion assay of i) MCF7 cells in NBF-conditioned medium and ii) MCF7 cells in CAF-conditioned medium. (B) Percentage of MCF7 cells invading through transwell while grown in CAF-conditioned medium compared with NBF-conditioned medium in CAF/NBF pairs \#2 and \#3. Results are average of two experiments done in duplicate. ${ }^{*} P$ value $=0.0021$, while ${ }^{\#} P$ value $=0.0011$.

Published by Bioscientifica Ltd. 
MCF7 cells, however, there was a significant decrease in expression of E-cadherin by western blotting in MCF7 cells co-cultured with CAFs compared with cells co-cultured with NBFs. Interestingly, Janda et al. found that with TGF $\beta$ treatment of RafER-EpH4 cells, E-cadherin protein levels were reduced $48 \mathrm{~h}$ after treatment while CDH1 mRNA levels were only reduced after 5 days of treatment. They concluded that loss of E-cadherin protein preceded transcriptional down-regulation of $C D H 1$ mRNA by over 2-3 days (Janda et al. 2006). We did not find consistent mRNA changes of the $C D H 1$ gene by qPCR even after 10 days of co-culture.

Lebret et al. (2007) studied the effect of co-culture of CAFs and NBFs from breast reduction specimens on PMC42-LA, a human breast cancer cell line with stem cell-like properties, which is able to exhibit either luminal epithelial or myoepithelial markers depending on the culture microenvironment. When PMC42-LA cells were co-cultured with CAFs and NBFs, the cells showed significantly increased expression of $\alpha$-SMA and cytokeratin 14 (myoepithelial markers) compared with control cells. There was also an increase in expression of vimentin in cells co-cultured with CAFs or NBFs compared with control. There was, however, no difference in expression of $\alpha$-SMA, cytokeratin 14 or vimentin in PMC42-LA cells co-cultured with CAFs or NBFs (Lebret et al. 2007). E-cadherin appeared to localise to cell-cell junctions in control PMC42-LA cells. When co-cultured with NBFs, E-cadherin was found in both junctional and cytoplasmic location while E-cadherin location in cells co-cultured with CAFs appeared to be more cytoplasmic. While we found less overall expression of E-cadherin in MCF7 cells co-cultured with CAFs compared with NBFs by immunofluorescence and confocal microscopy, we did not find a difference in intracellular localisation of E-cadherin in MCF7 cells co-cultured with CAFs or NBFs. Giannoni et al. studied the effects of conditioned medium of human prostate CAFs on PC3 cells, a cell line originating from bone metastasis of human prostate cancer cells. They found up-regulation of EMT markers Snail, Twist and vimentin and down-regulation of E-cadherin. There was also a change in morphology of the cells towards a spindle-shaped appearance, again consistent with EMT (Giannoni et al. 2010).

We found an increase in invasion of MCF7 cells grown in CAF-conditioned compared with NBF-conditioned medium. This is in keeping with a greater degree of EMT in MCF7 cells cultured in CAF- compared with NBF-conditioned medium.

\section{Conclusion}

Fibroblasts cultured from within breast cancer tissue are different to those cultured from macroscopically normal tissue located at least $2 \mathrm{~cm}$ away from the tumour margin. These CAFs have the ability to induce a greater degree of EMT in MCF7 breast cancer and MCF10A breast epithelial cell lines, indicating that CAFs are able to contribute to a more malignant breast cancer phenotype and their role in resistance to therapy should therefore be considered when treating breast cancer.

\section{Declaration of interest}

The authors declare that there is no conflict of interest that could be perceived as prejudicing the impartiality of the research reported.

\section{Funding}

This work was funded by the University of Sydney Cancer Research Fund. PS H Soon was also funded by National Health and Medical Research Council (Health Professional Research Training Fellowship \#511996) and New South Wales Cancer Institute (Clinical Research Fellowship \#09/CRF/2-05).

\section{Authors contribution statement}

P S H Soon conceived, designed and coordinated the study as well as drafted the manuscript. E Kim carried out cell culture, characterisation and EMT studies as well as data analysis. C K Pon carried out cell culture, EMT and flow cytometry studies as well as data analysis. A J Gill performed pathological review of breast tissue used in this study. $K$ Moore and A J Spillane collected tissue and revised the manuscript critically. D E Benn participated in the design of the study, provided intellectual input and critically revised the manuscript. R C Baxter conceived and designed the study as well as provided intellectual input and critically revised the manuscript. All authors read and approved the final manuscript.

\section{Acknowledgements}

The authors would like to acknowledge Lily Huschtscha for providing the protocol for primary culture of fibroblasts as well as fibroblasts from breast reduction specimens and Mark Sywak for collecting some of the tissue samples.

\section{References}

Anderberg C \& Pietras K 2009 On the origin of cancer-associated fibroblasts. Cell Cycle 8 1461-1462. (doi:10.4161/cc.8.10.8557)

Bhowmick NA, Neilson EG \& Moses HL 2004 Stromal fibroblasts in cancer initiation and progression. Nature 432 332-337. (doi:10.1038/ nature03096)

Blanco MJ, Moreno-Bueno G, Sarrio D, Locascio A, Cano A, Palacios J \& Nieto MA 2002 Correlation of Snail expression with histological grade and lymph node status in breast carcinomas. Oncogene 21 3241-3246. (doi:10.1038/sj.onc.1205416)

Brabletz T, Hlubek F, Spaderna S, Schmalhofer O, Hiendlmeyer E, Jung A \& Kirchner T 2005 Invasion and metastasis in colorectal cancer: epithelial-mesenchymal transition, mesenchymal-epithelial 
transition, stem cells and $\beta$-catenin. Cells, Tissues, Organs 179 56-65. (doi:10.1159/000084509)

Edge SB, Byrd DR \& Compton CC 2009 AJCC Cancer Staging Manual. New York, NY, USA: Springer.

Elenbaas B \& Weinberg RA 2001 Heterotypic signaling between epithelial tumor cells and fibroblasts in carcinoma formation. Experimental Cell Research 264 169-184. (doi:10.1006/excr.2000.5133)

Franco OE, Shaw AK, Strand DW \& Hayward SW 2010 Cancer associated fibroblasts in cancer pathogenesis. Seminars in Cell and Developmental Biology 21 33-39. (doi:10.1016/j.semcdb.2009.10.010)

Gao MQ, Kim BG, Kang S, Choi YP, Park H, Kang KS \& Cho NH 2010 Stromal fibroblasts from the interface zone of human breast carcinomas induce an epithelial-mesenchymal transition-like state in breast cancer cells in vitro. Journal of Cell Science 123 3507-3514. (doi:10.1242/ jcs.072900)

Giannoni E, Bianchini F, Masieri L, Serni S, Torre E, Calorini L \& Chiarugi P 2010 Reciprocal activation of prostate cancer cells and cancerassociated fibroblasts stimulates epithelial-mesenchymal transition and cancer stemness. Cancer Research 70 6945-6956. (doi:10.1158/ 0008-5472.CAN-10-0785)

Gregory PA, Bert AG, Paterson EL, Barry SC, Tsykin A, Farshid G, Vadas MA, Khew-Goodall Y \& Goodall GJ 2008 The miR-200 family and miR-205 regulate epithelial to mesenchymal transition by targeting ZEB1 and SIP1. Nature Cell Biology 10 593-601. (doi:10.1038/ncb1722)

Hawsawi NM, Ghebeh H, Hendrayani SF, Tulbah A, Al-Eid M, Al-Tweigeri T, Ajarim D, Alaiya A, Dermime S \& Aboussekhra A 2008 Breast carcinoma-associated fibroblasts and their counterparts display neoplastic-specific changes. Cancer Research 68 2717-2725. (doi:10.1158/0008-5472.CAN-08-0192)

Hayward SW, Wang Y, Cao M, Hom YK, Zhang B, Grossfeld GD, Sudilovsky D \& Cunha GR 2001 Malignant transformation in a nontumorigenic human prostatic epithelial cell line. Cancer Research 61 8135-8142.

Holliday DL, Brouilette KT, Markert A, Gordon LA \& Jones JL 2009 Novel multicellular organotypic models of normal and malignant breast: tools for dissecting the role of the microenvironment in breast cancer progression. Breast Cancer Research 11 R3. (doi:10.1186/ bcr2218)

Huang M, Li Y, Zhang H \& Nan F 2010 Breast cancer stromal fibroblasts promote the generation of CD44+CD24 - cells through SDF-1/CXCR4 interaction. Journal of Experimental \& Clinical Cancer Research 2980. (doi:10.1186/1756-9966-29-80)

Janda E, Nevolo M, Lehmann K, Downward J, Beug H \& Grieco M 2006 Raf plus TGFß-dependent EMT is initiated by endocytosis and lysosomal degradation of E-cadherin. Oncogene 25 7117-7130. (doi:10.1038/ sj.onc.1209701)

Kajiyama H, Shibata K, Terauchi M, Yamashita M, Ino K, Nawa A \& Kikkawa F 2007 Chemoresistance to paclitaxel induces epithelial-mesenchymal transition and enhances metastatic potential for epithelial ovarian carcinoma cells. International Journal of Oncology 31 277-283.

Kalluri R \& Weinberg RA 2009 The basics of epithelial-mesenchymal transition. Journal of Clinical Investigation 119 1420-1428. (doi:10.1172/ JCI39104)

Lakhani SR, Ellis IO, Schnitt SJ, Tan PH \& van de Vijver MJ 2012 In WHO Classification of Tumours of the Breast. Lyon, France: IARC.

Lebret SC, Newgreen DF, Thompson EW \& Ackland ML 2007 Induction of epithelial to mesenchymal transition in PMC42-LA human breast carcinoma cells by carcinoma-associated fibroblast secreted factors. Breast Cancer Research 9 R19. (doi:10.1186/bcr1656)

Mishra PJ, Humeniuk R, Medina DJ, Alexe G, Mesirov JP, Ganesan S, Glod JW \& Banerjee D 2008 Carcinoma-associated fibroblast-like differentiation of human mesenchymal stem cells. Cancer Research 68 4331-4339. (doi:10.1158/0008-5472.CAN-08-0943)

Mueller MM \& Fusenig NE 2004 Friends or foes - bipolar effects of the tumour stroma in cancer. Nature Reviews. Cancer 4 839-849. (doi:10.1038/nrc1477)

Olumi AF, Grossfeld GD, Hayward SW, Carroll PR, Tlsty TD \& Cunha GR 1999 Carcinoma-associated fibroblasts direct tumor progression of initiated human prostatic epithelium. Cancer Research 59 5002-5011.

Sonnenberg M, van der Kuip H, Haubeis S, Fritz P, Schroth W, Friedel G, Simon W, Murdter TE \& Aulitzky WE 2008 Highly variable response to cytotoxic chemotherapy in carcinoma-associated fibroblasts (CAFs) from lung and breast. BMC Cancer 8 364. (doi:10.1186/ 1471-2407-8-364)

Sporn MB 1996 The war on cancer. Lancet 347 1377-1381. (doi:10.1016/ S0140-6736(96)91015-6)

Storci G, Sansone P, Trere D, Tavolari S, Taffurelli M, Ceccarelli C, Guarnieri T, Paterini P, Pariali M, Montanaro L et al. 2008 The basal-like breast carcinoma phenotype is regulated by SLUG gene expression. Journal of Pathology 214 25-37. (doi:10.1002/path.2254)

Tlsty TD \& Hein PW 2001 Know thy neighbor: stromal cells can contribute oncogenic signals. Current Opinion in Genetics \& Development 11 54-59. (doi:10.1016/S0959-437X(00)00156-8)

Wald O, Izhar U, Amir G, Kirshberg S, Shlomai Z, Zamir G, Peled A \& Shapira OM 2011 Interaction between neoplastic cells and cancerassociated fibroblasts through the CXCL12/CXCR4 axis: role in non-small cell lung cancer tumor proliferation. Journal of Thoracic and Cardiovascular Surgery 141 1503-1512. (doi:10.1016/j.jtcvs. 2010.11.056)

Yang AD, Fan F, Camp ER, van Buren G, Liu W, Somcio R, Gray MJ, Cheng H, Hoff PM \& Ellis LM 2006 Chronic oxaliplatin resistance induces epithelial-to-mesenchymal transition in colorectal cancer cell lines. Clinical Cancer Research 12 4147-4153. (doi:10.1158/1078-0432.CCR06-0038)

Received in final form 24 October 2012

Accepted 25 October 2012

Made available online as an Accepted Preprint

30 October 2012 http://www.erc.endocrinology-journals.org DOI: 10.1530/ERC-12-0227
(C) 2013 Society for Endocrinology Printed in Great Britain
Published by Bioscientifica Ltd. 